\title{
A loopy view
}

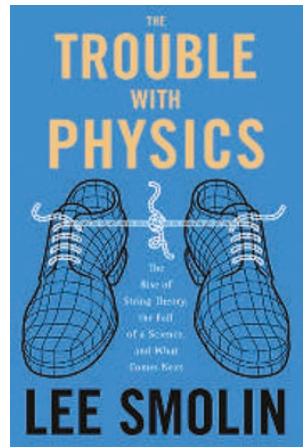

\section{THE TROUBLE WITH PHYSICS: THE RISE OF STRING THEORY, THE FALL OF SCIENCE AND WHAT COMES NEXT BY LEE SMOLIN}

Houghton Mifflin Company: 2006. 416 pp. \$26.00 (pbk)

"I think of myself as a seer", writes Lee Smolin in this no-holds-barred attack on the science and sociology of string theory. "Why are string theorists not seers?" In my opinion, toe-curling utterances such as these (of which there are many) are likely to backfire and leave the reader rooting for strings by the end of the book. The author says, "I personally dislike conflict and confrontation", but his book cover claims that as a scientific theory, string theory has been "a colossal failure" and is "dragging down the rest of science".

Now there is no doubt that superstring theory, and its successor M-theory, are the dominant paradigms in current attempts to unify gravity and quantum mechanics and to explain all physical phenomena. In terms of the number of its adherents, the jobs and prizes it accrues and the publicity it receives, string theory leaves all rival unified theories in its wake. So the frustration of those like Smolin who march to the beat of a different drum, such as 'loop quantum gravity', is understandable. To make matters worse, there is some truth in his complaints that as a community, string theorists are arrogant, exclusive and unwilling to listen to unorthodox views.

Smolin provides three pieces of historical evidence from the 1980s that string theory sociology leaves much to be desired: (1) the claim to be free of the infinities that plague quantum versions of Einstein's general relativity, though very plausible, had not actually been proven; (2) the claim of uniqueness of its equations obscured the fact that there were five different superstring theories and $10^{1500}$ different ways of rolling up the extra unobserved space-time dimensions; and (3) the claim that one-dimensional strings moving in a ten-dimensional world provided the one path to truth, ignored the strong evidence that higher dimensional branes and eleven space-time dimensions had a part to play in the grand scheme of things.

Subsequent developments have vindicated all three objections. Finiteness has still not been rigorously proven, but the billions of different compactifications have now become a feature of the 'multiverse' and the 'string landscape' picture, and the $1995 \mathrm{M}$-theory that unifies the five different superstrings is an eleven-dimensional theory involving branes. As a matter of fact, in 1987 I wrote my own critique of superstring orthodoxy raising precisely these three points, so I can certainly agree with Smolin that the stubbornness of string theorists and their follow-my-leader 'groupthink' have slowed down progress, even if I am less impressed with his originality in picking examples. But Smolin goes much further by denying that any progress has been made!

This, in my view, is the main weakness of the book. Smolin conflates sociology with science and concludes that because string enthusiasts are not his kind of people, and don't invite him to their meetings, their physics must be wrong. It is undeniable that string theory has not yet passed the test of experimental confirmation, but there are lots of examples in the history of science where good theoretical ideas waited a long time for experiment. Bose-Einstein condensation was predicted in 1925, but not confirmed until 1995; Yang and Mills invented non-abelian gauge bosons in 1954, but the W-boson was not discovered until 1982. Yet Smolin makes the sweeping claim that "before the 1970s, theory and experiment had developed hand-in-hand. New ideas were tested within a few years, ten at most". If we are to reject the $1995 \mathrm{M}$-theory because its ten years without definitive empirical proof are already up, then we must also reject gravitational waves (1916), the cosmological constant (1917), extra dimensions (1926), the Higgs boson (1964), supersymmetry (1971) and, er, loop quantum gravity (1986).

\section{Michael Duff}

Michael Duff is Abdus Salam Professor of Theoretical Physics at Imperial College London, Prince Consort Road, London SW7 2AZ, UK.
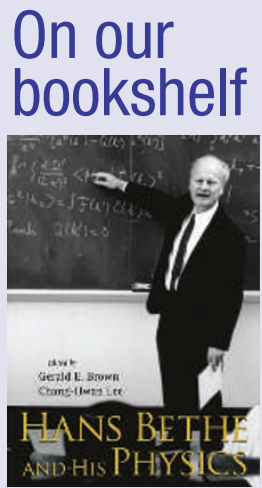

Hans Bethe and his Physics

Edited by

Gerald E. Brown and

Chang-Hwan Lee

World Scientific: 2006.

328 pp. £56.

Ranging from solidstate physics to nuclear physics to astrophysics, and spanning nearly eight decades, the work of Hans Bethe inspires a full team to explain Bethe's science and lasting legacy.

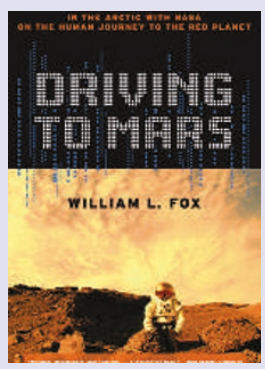

Driving to Mars: In the Arctic with NASA on the Human Journey to the Red Planet by William L. Fox Shoemaker \& Hoard: 2006. 272 pp. \$16.

Devon Island, in the Canadian arctic, features a "crater, water gullies, meltwater channels, and caves with perennial ice" an ideal environment for scientists preparing for an encounter with Mars. 